Dear Author,

Please, note that changes made to the HTML content will be added to the article before publication, but are not reflected in this PDF.

Note also that this file should not be used for submitting corrections. 


\title{
Determination of the constitutive relation and critical condition for the shock compression of cellular solids
}

\author{
Yongle Sun ${ }^{a}$, Q.M. Li ${ }^{\mathrm{a}, \mathrm{c}, *}$, S.A. McDonald ${ }^{\mathrm{b}}$, P.J. Withers ${ }^{\mathrm{b}}$ \\ a School of Mechanical, Aerospace and Civil Engineering, The University of Manchester, Sackville Street, Manchester M13 9PL, UK \\ ${ }^{\mathrm{b}}$ Henry Moseley X-ray Imaging Facility, School of Materials, The University of Manchester, Upper Brook Street, Manchester, M13 9PY, UK \\ ' State Key Laboratory of Explosion Science and Technology, Beijing Institute of Technology, Beijing 100081, China
}

\section{A R T I C L E I N F O}

\section{Article history:}

Received 19 August 2015

Revised 18 March 2016

Available online $\mathrm{xxx}$

\section{Keywords:}

Foam material

Compressive behaviour

Shock

Hugoniot

Computed tomography

\begin{abstract}
A B S T R A C T
This study aims at understanding the constitutive relation and critical condition for the shock compression of cellular solids. A 2D virtual foam is constructed from the cross-section of a closed-cell aluminium foam imaged by micro X-ray computed tomography, which enables the realistic consideration of mesoscale structural effect in numerical modelling. Quasi-static and shock compressions of the 2D foam are simulated. A series of Hugoniot relations between shock speed (and other mechanical quantities) and impact speed are determined from the FE simulations. It is found that the shock speed increases approximately linearly with impact speed, similar to that observed for condensed solids, but the related material constants for cellular solids have different physical implications, whereas the shock strain, stress and energy increase with impact speed nonlinearly, due to shock-enhanced cell compaction and cell-wall deformation. Based on conservation laws in continuum mechanics, other Hugoniot relations are derived from the basic linear one, which agree well with those obtained from FE simulations. It is thus demonstrated that the unique linear Hugoniot relation can be used to characterise the shock constitutive behaviour which is distinct from the quasi-static one. Furthermore, a new analytical method based on the linear Hugoniot relation is proposed to estimate the critical impact speed for shock initiation, which has reasonable agreement with the present FE simulation and previous experimental and numerical results, and outperforms the existing methods.
\end{abstract}

(C) 2016 Elsevier Ltd. All rights reserved.

\section{Introduction}

Cellular solids are characterised by high porosity (usually exceeding 70\%), leading to their distinctive mechanical, thermal, electromagnetic and other properties attractive for various engineering applications (Gibson and Ashby, 1997). Under high speed impact or intensive blast, shock compression can be initiated in cellular solids and the load transmitted can be significantly increased (Elnasri et al., 2007; Li and Meng, 2002; Reid and Peng, 1997; Tan et al., 2012; Tan et al., 2005a), which may enhance the energy absorption but not benefit structural protection. Therefore, it is important to understand the shock behaviour of cellular solids.

Extensive experiments have identified two prominent features of shock compression in cellular solids (Barnes et al., 2014; Radford et al., 2005; Reid and Peng, 1997; Tan et al., 2012; Tan et al., 2005a): (1) a significant enhancement of stress measured at the impact end; and (2) a localisation of cell crushing adjacent to the

\footnotetext{
* Corresponding author.

E-mail address: qingming.li@manchester.ac.uk (Q.M. Li).
}

impact end. Meanwhile, numerical studies on the dynamic crushing of idealised 2D cellular solids have been reported to understand the effects of cell irregularity (Zheng et al., 2005), nonuniform cell-wall thickness (Li et al., 2007), cell micro-topology (Liu and Zhang, 2009) and structural defects (Zhang et al., 2010) on the shock behaviour. However, Sun et al. (2015; 2016b) have demonstrated the importance to use realistic cell geometry in the simulation of dynamic crushing in order to capture the distinctive shock behaviour. Therefore, it is necessary to apply more realistic cellular solid in modelling.

"Shock", as one of the deformation modes of cellular solids, is used here as a term for the propagation of the planar interface (i.e. shock front) separating the crushed and uncrushed cells in dynamic compression, which has similar feature to shock wave propagation in a condensed solid (Davison, 2008; Meyers, 1994; Wang, 2007). To demonstrate the "shock" feature of the shock front, Zou et al. (2009) performed finite element simulations and numerically observed a jump or macroscopic discontinuity in the key mechanical quantities (i.e. velocity, stress and strain) across the shock front (about one cell size in thickness) of hexagonal-cell 
honeycombs. Liao et al. (2013) numerically confirmed the existence of shock front for idealised irregular 2D cellular structures.

The measurement of the speed of shock front (i.e. shock speed for brevity) has been also attempted in experiments and simulations. For instance, Barnes et al. (2014) experimentally observed that the variation of shock speed with impact speed generally follows a linear trend for open-cell aluminium Duocel foam and a linear equation was used to fit the experimental data. Liao et al. (2013) derived the relation between shock speed and impact speed for 2D Voronoi foam based on various idealisations of the quasi-static stress-strain relation; but their analytical predictions showed marked difference from the numerical result, especially at high impact speeds (see Fig. 13 in Ref. (Liao et al., 2013)). Similarly, Pattofatto et al. (2007) compared the analytical predictions of shock speed derived from the quasistatic stress-strain relation for closed-cell aluminium Alporas foam and they stated that their nonlinear equation (see Eq. 9 in Ref. (Pattofatto et al., 2007)) gave a "satisfactory prediction". More recently, Zheng et al. (2014) measured the dynamic stress-strain relation for 3D Voronoi foam numerically and recommended a dynamic material model, i.e. Eq. (14) in Ref. (Zheng et al. (2014)), based on which, we found that a linear relation between shock speed and impact speed, i.e. $\dot{\Phi}=v+\sqrt{D / \rho_{0}}$, can be derived (see Appendix) where $\dot{\Phi}$ is the shock speed, $v$ is the impact speed, $\rho_{0}$ is the density and $D$ is a material constant defined therein. The simple linear relation between shock speed and impact speed seems more fundamental for characterising and modelling the shock behaviour of cellular solids. However, it requires more experimental and numerical supports and a better understanding of its physical implications.

On the other hand, increasing efforts have been made to establish the constitutive relation under shock compression. In the early study, Reid and Peng (1997) proposed a shock model based on a rigid, perfectly-plastic, locking ( $\mathrm{r}-\mathrm{p}-\mathrm{p}-\mathrm{l})$ material model to analyse the impact response of wood, which was subsequently adopted by other researchers to analyse the shock behaviour of various cellular solids (Li and Reid, 2006; Ma et al., 2009; Ruan et al., 2003; Tan et al., 2005b; Zou et al., 2009). The continuous improvement of this shock model is mainly from the adoption of more realistic constitutive equations such as linear hardening (Zheng et al., 2012), power-law hardening (Zheng et al., 2013) and complicated nonlinear hardening (Karagiozova et al., 2012) equations. However, the basic assumption that the shock state of the material is independent of the local crushing velocity has not been changed. In other words, the quasi-static compression tests were considered to be able to provide complete input parameters for the shock model. Recently this assumption has been proven questionable. For instance, Zheng et al. (2014) employed a 3D Voronoi finite element model to establish a dynamic stress-strain relation which is shown different from the quasi-static one. Barnes et al. (2014) experimentally measured the mechanical variables of the Duocel foam at different impact speeds and demonstrated that a complete description of shock behaviour requires the direct measurement of Hugoniot relations (i.e. the loci of all shocked states) and the material states under shock cannot be determined from the quasi-static stress-strain relation. These recent findings do not support the conclusion made by Pattofatto et al. (2007) that "shock enhancement effect should not be taken into account at the level of the constitutive law itself".

Equally importantly, previous experimental and numerical studies have shown that there exists a critical impact speed, above which shock occurs (Barnes et al., 2014; Tan et al., 2005a; Zou et al., 2009). For compression at subcritical impact speeds, localised cell crushing occurs in presumably "weak" cells or sites, and the crush bands are randomly distributed and the boundary between crushed and uncrushed cells is not necessarily flat (Barnes et al., 2014; Liu et al., 2009; Sun et al., 2014; Tan et al., 2005a; Zheng et al., 2014), in contrast to the shock deformation mode. The critical condition for shock initiation is of fundamental importance and practical interest. However, it still lacks a recognised analytical method to determine this critical impact speed, and there is confusion about the factors that influence the critical impact speed (Wang et al., 2013).

The objective of this study is to clarify above outstanding issues. A combined image-based modelling and continuum-based theory were applied. A 2D virtual foam was created from the computed tomography (CT) image of a cross-section of a closed-cell aluminium Alporas foam sample and a finite element (FE) model with the same meso-scale complexity of the real foam was developed for compression simulations. First, the quasi-static compression of the 2D foam was simulated for comparison purpose. Then the shock compression was simulated at different impact speeds to obtain the complete Hugoniot relations (e.g. the dependences of shock speed and material states on impact speed, and the stressstrain relation) and to define the shock constitutive relation. Furthermore, a new analytical method based on the basic Hugoniot relation (shock speed vs. impact speed) is proposed to estimate the critical impact speed for shock initiation.

\section{Image-based modelling}

\subsection{Two-dimensional foam}

A diametral X-ray computed tomography (CT) slice image of a cylindrical sample $(\varnothing 30 \times 30 \mathrm{~mm})$ of closed-cell aluminium Alporas foam was used to construct a $2 \mathrm{D}$ virtual foam $(30 \times 30 \mathrm{~mm})$ with realistic cell geometry, as shown in Fig. 1. The CT scanning of the real foam sample was performed in a Nikon Metris CT system housed in a customised bay at the Henry Moseley X-ray Imaging Facility (HMXIF, The University of Manchester). An acceleration voltage of $70 \mathrm{kV}$, a current of $280 \mu \mathrm{A}$, an effective voxel size of $19.1 \mu \mathrm{m}$, and an exposure time of $500 \mathrm{~ms}$ for each of 2000 projections over 360 degrees were used. The X-ray radiographs were reconstructed using Nikon Metris CT-Pro software into CT images. A greyscale-based segmentation method was then used to extract the cell structure of the foam, which adopted a threshold of grey values to ensure the separation of all the solid parts from the surrounding air.

It is clearly seen from Fig. 1 that the cell morphology and topology of the 2D virtual foam are much more complex than those of the idealised 2D cellular solids such as hexagonal-cell, circular-cell and Voronoi ones (Liu et al., 2009; Sun and Li, 2015; Zheng et al., 2005). Some structural imperfections, e.g. corrugation, bowed or damaged walls, and non-uniform cell-wall thickness, are evident, which are related to the liquid-state foaming process of Alporas foam (Simone and Gibson, 1998a). These kinds of structural defects may play important roles in the determination of macroscopic material properties (Chen et al., 1999; McDonald et al., 2006; Simone and Gibson, 1998b, 1998c). However, due to the difficulty to control the variation of the structural imperfection in the selected sample, the influence of structural imperfection will not be the focus of this study.

To take account of realistic geometrical features, continuum elements have to be used in a finite element model (FEM), which dramatically increases the computational expense and may also cause numerical problems due to element distortion. The 3D FE modelling of the compressive behaviour of Alporas foam has been attempted by the same authors (Sun et al., 2016b). However, the 3D FE simulation is still restricted to relatively small samples and less intensive deformations for limited loading cases
102 
(a)

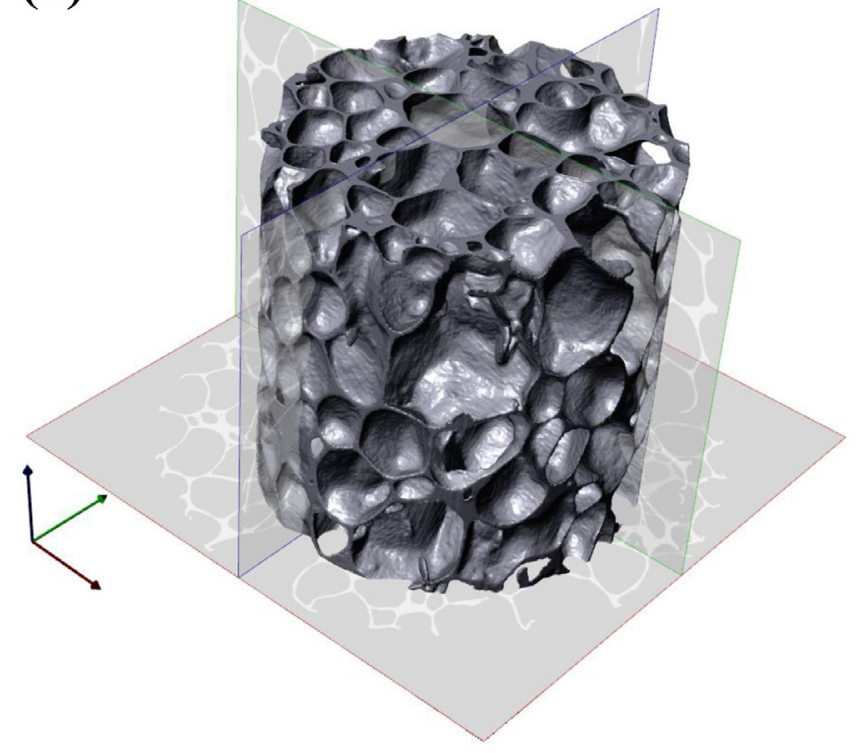

(b)

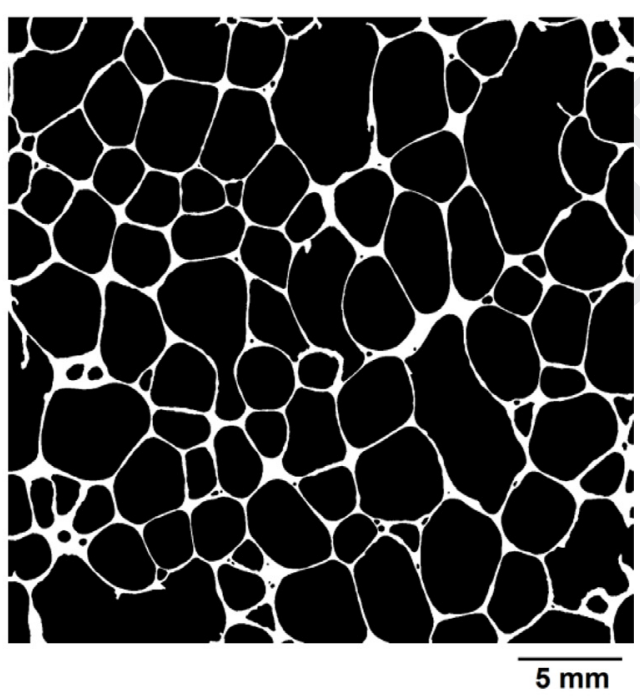

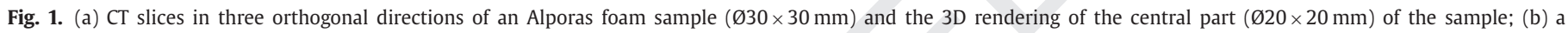
binarised diametral slice corresponding to an actual cross-section of the sample.

Table 1

Structural parameters of the 2D foam.

\begin{tabular}{lllll}
\hline & Average & $\begin{array}{l}\text { Standard } \\
\text { deviation }\end{array}$ & Maximum & Minimum \\
\hline Cell size $(\mathrm{mm})$ & 2.05 & 1.60 & 7.48 & 0.09 \\
Cell-wall thickness $(\mathrm{mm})$ & 0.36 & 0.42 & 1.06 & 0.04 \\
\hline
\end{tabular}

(Jeon et al., 2010; Maire and Withers, 2014; McDonald et al., 2011; Sun et al., 2016a; Sun et al., 2016b; Sun et al., 2014), which hinders the analysis of shock compression related to extreme cell crushing and complex cell-wall contact at high speed impact. Therefore, the present study focuses on the shock compression of the 2D virtual foam constructed from the CT slice image.

As the 2D foam is based on an actual cross-section taken from its 3D counterpart, it may be regarded as a 2D "replica" of closedcell aluminium foams having similar cross-sectional geometry to Alporas foam. However, it should be noted that 3D foams have more complex geometrical elements (e.g. cell faces) and deformation modes (e.g. membrane stretch). This fact must be borne in mind as the analysis using the 2D foam model is essentially qualitative to provide general insights. But it has advantages over the idealised foam models (e.g. Hönig and Stronge, 2002; Liu et al., 2009; Ma et al., 2009) in the sense of realistically incorporating the existing structural defects.

The $2 \mathrm{D}$ foam has a relative density ( $\mathrm{RD}$, i.e. area ratio between cell walls and foam sample) of $12.5 \%$. The size for each cell is obtained as the diameter of a circle having the same area as the cell and the average cell size is $2.05 \mathrm{~mm}$. There are 148 cells in total, of which 111 are inside the sample and 37 are located on the boundaries. The local cell-wall thickness at a given material point (pixel in the digital image) is defined as the diameter of the largest circle which contains the point and fits completely inside the wall (Hildebrand and Rüegsegger, 1997), and the 2D foam has an average cell-wall thickness of $0.36 \mathrm{~mm}$. The structural parameters are summarised in Table 1 . Besides the large scatter of cell size and cell-wall thickness, the cell shape also exhibits a wide variety (see Fig. 1b), indicating the heterogeneous nature of the cell structure at the meso-scale.

\subsection{Finite element model}

The meshing of the 2D foam structure obtained from the CT slice image (see Fig. 1b) was accomplished by using ScanIP (Simpleware Ltd, UK), which is designed to directly convert CT image into high-quality FE mesh (Young et al., 2008). A total number of 398,703 linear elements with reduced integration were used, as shown in Fig. 2. The FE solver Abaqus/Explicit was employed for the numerical simulations. The large deformation effect was incorporated and the plane strain condition was assumed. Herein, the cell-wall yield strength reported for Alporas foam, i.e. $172 \mathrm{MPa}$ (Simone and Gibson, 1998a), was adopted in a rate-independent perfect plasticity material model. The elastic modulus, Poisson's ratio and density were taken to be $68 \mathrm{GPa}, 0.33$ and $2710 \mathrm{~kg} / \mathrm{m}^{3}$, respectively.

The loading was applied across the two ends of the 2D foam sample by two rigid platens (not shown in Fig. 2). During loading, the top platen moved downward with a constant speed (varied in different loading cases and denoted as $V_{\mathrm{i}}$ ), while the bottom platen was fixed to support the sample. Frictionless contact was considered between the platens and cell walls, as well as between the cell walls themselves. No direct constraints were applied to the foam. Under such loading, the foam was subjected to uniaxial compression macroscopically. In order to visualise and quantify the heterogeneity of the deformation, we used a "particle" array approach as illustrated in Fig. 2 where a vertical line of 79 "particles" was used to represent the average motion of 79 equally spaced cross-sections through the foam (excluding the two end surfaces), which is similar to the method proposed by Zou et al. (2009).

The nominal (engineering) compressive stresses measured at the two sample ends are obtained as the reaction forces at the corresponding rigid platens divided by the original width of the sample. The nominal (engineering) compressive strain is defined as the ratio of the vertical displacement of the loading platen to the original sample height. The shock strain for the crushed zone behind shock front is defined over the original height of the shocked cells, of which the determination method will be elaborated later when presenting the relevant numerical results. 


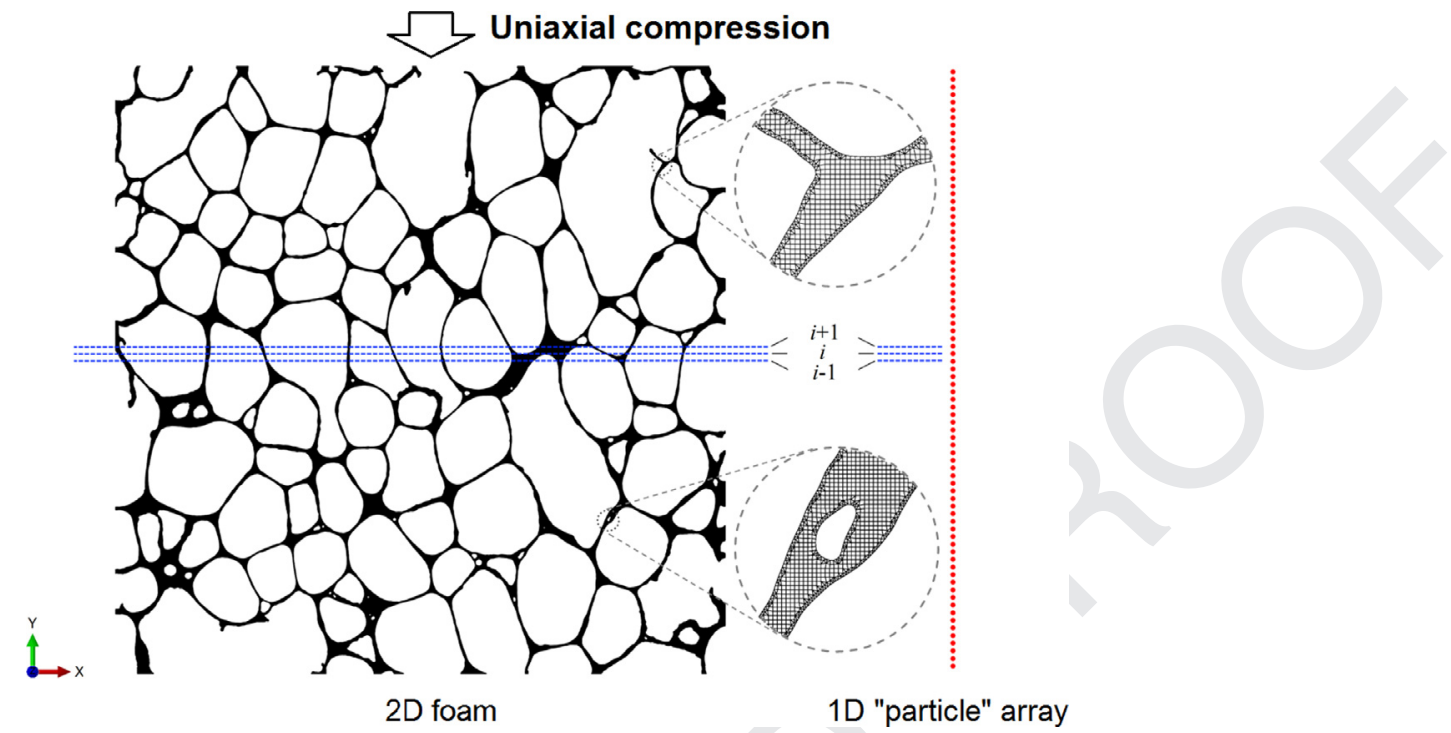

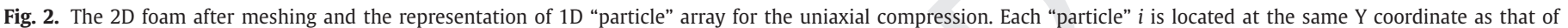
the corresponding transverse cross-section $i$ of the undeformed 2D foam and the "particle" motion represents the average of the FE nodal motion of the cross-section.

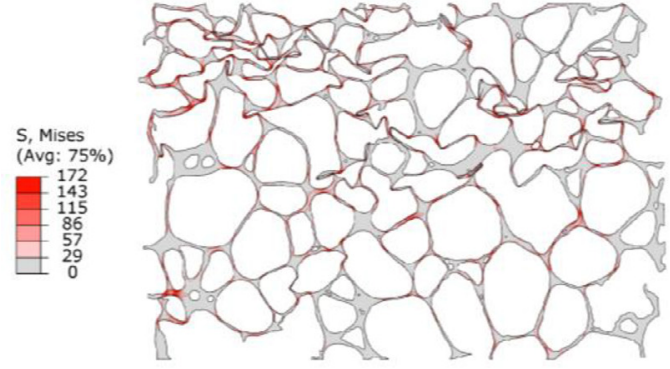

(a)

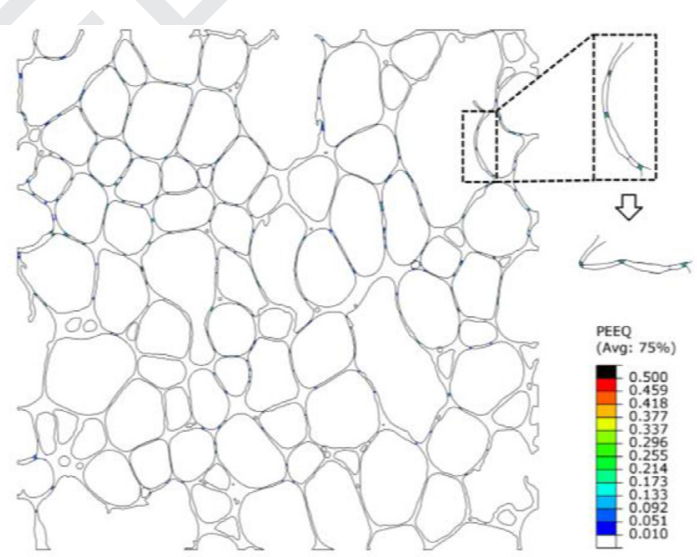

(b)

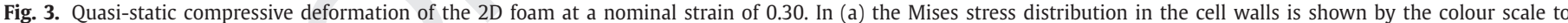

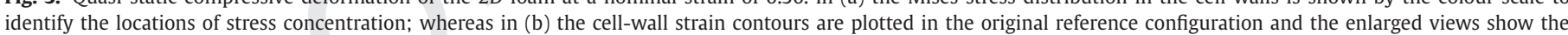

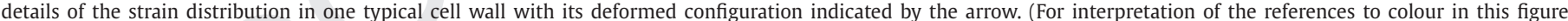
legend, the reader is referred to the web version of this article.)

\section{Results}

\subsection{Quasi-static compression}

The compression at a quasi-static strain-rate $\left(1 \times 10^{-3} \mathrm{~s}^{-1}\right)$ was simulated first, wherein inertia effect is negligible. The nominal stress-strain curve obtained from the 2D compression simulation, as shown in Fig. 4, captures the key characteristics of the quasistatic stress-strain relation of cellular solids, i.e. the stress increases to a local maximum and then drops due to cell collapse after which the stress remains more or less constant leading to a plateau regime followed by a rapid stress rise due to foam densification.

Fig. 3 shows the quasi-static compressive deformation of the 2D foam at a nominal strain of 0.30 . A crush band (somewhat inclined) forms near the top end. The cell-wall stress is mainly concentrated within the crush band, as seen from the Mises stress distributions shown in Fig. 3a. The plastic buckling and bending of cell walls, as a primary collapse mode, contribute most to the nominal deformation. Plastic hinges are prevalent in the cell walls, as shown in Fig. $3 \mathrm{~b}$.

\subsection{Shock compressive stress and deformation}

With the increasing of the loading speed the inertia effect becomes important, which can lead to a significant imbalance between the reaction forces at the impact and support ends. Fig. 4 shows the comparison between the quasi-static and dynamic compressive stress-strain curves. At a moderate impact speed (e.g. $V_{\mathrm{i}}=$ $18 \mathrm{~m} / \mathrm{s}$ ), the nominal stresses at the impact and support ends are close and the dynamic stress-strain curve is similar to the quasistatic one, suggesting that the inertia effect is insignificant in this case. By contrast, when the impact speed further increases (e.g. $V_{\mathrm{i}}=90 \mathrm{~m} / \mathrm{s}$ ), the stress magnitude at the impact end becomes significantly larger than both the supporting stress and the quasistatic stress, indicating that in such a loading case the global nominal stress-strain relation cannot describe the constitutive compressive behaviour. It should be also noted that a severe stress fluctuation is observed at the high speed impact, which partly results from the contact interaction and the numerical solution algorithm (Abaqus/Explicit) used in the FE simulation, as discussed in Refs. (Zheng et al., 2014; Zou et al., 2009). The stress enhancement at 


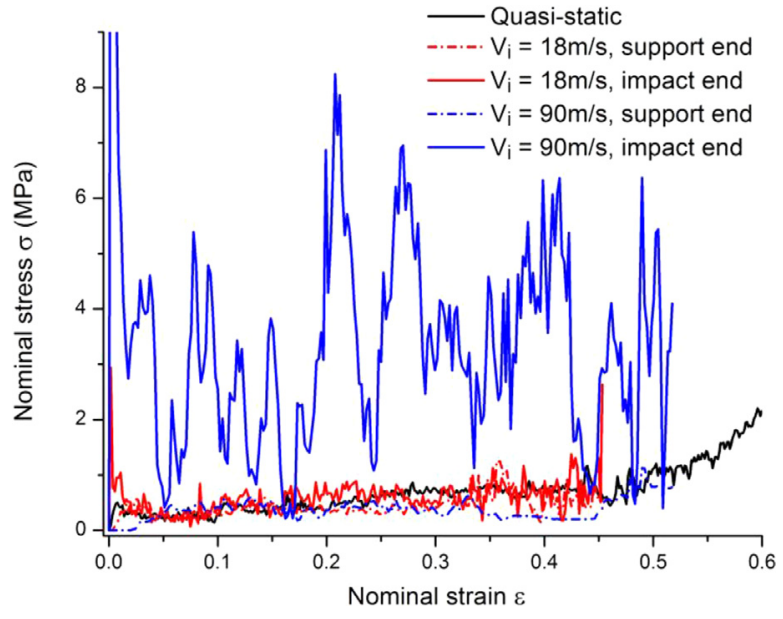

Fig. 4. Comparison of the quasi-static and dynamic stress-strain curves. the impact end has been generally recognised as a result of shock effect (see Section 1).

The cell deformation under shock compression, as shown in Fig. 5, is distinct from that under quasi-static compression (see (e.g. $V_{\mathrm{i}}=18 \mathrm{~m} / \mathrm{s}$ ) is not shown here since it is similar to the quasi-static one. Under shock compression, the cell deformation is concentrated at the impact end, from which the cell crushing propagates towards the support end in a layer-by-layer manner, and the shock deformation is intensified when impact speed increases, as shown in Fig. 5. This is consistent with previous numerical and experimental observations, as introduced in Section 1. It is also noteworthy that the lateral deformation under shock is negligible, despite the cell compaction in the crushed zone.

To examine the cell-wall deformation at different impact speeds, the plastic strain distribution in cell walls is also shown in Fig. 5. It is clearly seen that plastic hinges are widely spread in the cell walls, resulting from the plastic bending and buckling of the cell walls. In general, the junction portions are less deformed and the plastic deformation is mainly distributed in thin walls. Furthermore, when impact speed increases more plastic hinges are produced in the cell walls, which has significant implication for energy absorption of foam materials under shock compression and will be further discussed later.

Fig. 6 shows the distributions of uniaxial cross-sectional velocity. Although the original cross-section becomes uneven when cells are crushed, as shown in Fig. 5, it is expected that the cell compaction reduces the velocity variation between different material points in the crushed zone. Therefore, the velocity of the original cross-section is used to indicate the average of the vertical velocities of the material points located within each cross-section. It is evident that the cross-sectional velocities in the crushed zone (adjacent to the top end) are close to the impact speed, while those in the uncrushed zone (adjacent to the bottom end) are almost zero. Consequently there is a jump in the velocity distribution, which defines a shock front as shown in Fig. 5. However, the shock front, which represents a macroscopic discontinuity, actually has a finite thickness within which the material velocity changes rapidly but continuously at the meso-scale. This thickness is at least one cell size for the 2D foam with heterogeneous cell structure. A similar observation has also been reported by Zou et al. (2009) for regular hexagonal-cell honeycombs.

Based on the cross-sectional velocity profile, the determination of the position of the shock front can be implemented. The position of the shock front is determined at the cross-section having a Fig. 3). Note that the cell deformation at moderate impact speeds velocity drop of approximately a half of the impact velocity, which is able to have the resolution limited to one average cell size, since the shock front usually has a thickness of at least one average cell size.

\subsection{Shock speed and material states}

To obtain the shock speed $V_{s}$, the variations of the position of shock front with the time are measured for different impact speeds, as shown in Fig. 7a. It is clearly seen that the position of the shock front is approximately linearly dependent on loading time, and thus the shock speed can be obtained as the slope of the linear fitting line, as suggested in Refs. (Elnasri et al., 2007; Liao et al., 2013). The variation of shock speed with impact speed is shown in Fig. $7 \mathrm{~b}$, from which a linear relation is evident, and a

$V_{\mathrm{S}}=V_{\mathrm{r}}+S V_{\mathrm{i}}$

where $V_{\mathrm{r}}$ is a reference speed ( $V_{\mathrm{s}}$ intercept), $V_{\mathrm{i}}$ is the impact speed and $S$ is an material parameter. Here we have $V_{r}=20.16 \mathrm{~m} / \mathrm{s}$ and $S=1.135$ according to Fig. $7 \mathrm{~b}$.

As the cell crushing of the 2D foam under shock compression propagates in a similar manner to the 1D shock wave in a continuum medium, 1D conservation laws for a continuum solid can be used to establish the governing equations for the shock compression of the 2D foam. The conservation equations of mass, momentum and energy in a continuum solid wherein a 1D shock wave propagates are (Wang, 2007)

$V_{\mathrm{b}}-V_{\mathrm{a}}=V_{\mathrm{s}}\left(\varepsilon_{\mathrm{b}}-\varepsilon_{\mathrm{a}}\right)$

$\sigma_{\mathrm{b}}-\sigma_{\mathrm{a}}=\rho_{0} V_{\mathrm{s}}\left(V_{\mathrm{b}}-V_{\mathrm{a}}\right)$

$U_{\mathrm{b}}-U_{\mathrm{a}}=\frac{1}{2}\left(\sigma_{\mathrm{b}}+\sigma_{\mathrm{a}}\right)\left(\varepsilon_{\mathrm{b}}-\varepsilon_{\mathrm{a}}\right)$

where $V, \varepsilon, \sigma$ and $U$ are material velocity, engineering strain, engineering stress and internal energy density, respectively; $\rho_{0}$ is the initial density; the subscript $\mathrm{s}$, a and b denotes the shock front, the material ahead of the shock front and the material behind the shock front, respectively. Note that in the above equations it is assumed that the 2D foam has been homogenised. In addition, as the cell-wall material is assumed to be rate-independent and have no other irreversible processes (e.g. heating and friction) involved except plastic dissipation in the modelling, the internal energy is equal to strain energy in this case.

Fig. 8 illustrates the shock propagation and the material states across the shock front. Since the material ahead of the shock front is uncrushed (almost "undeformed", see Fig. 5), their deformation state can be approximated as $\varepsilon_{\mathrm{a}} \approx 0$ (i.e. $h_{\mathrm{a}} \approx H_{\mathrm{a}}$ ) and $V_{\mathrm{a}} \approx 0 \mathrm{~m} / \mathrm{s}$. From this approximation, we further have $U_{\mathrm{a}} \approx 0 \mathrm{~J} / \mathrm{m}^{3}$, but the stress $\sigma_{\mathrm{a}}$ still has to be determined since negligible deformation does not necessarily lead to negligible stress (see Fig. 4). For the material behind the shock front, the only approximation can be made is that $V_{\mathrm{b}} \approx V_{\mathrm{i}}$ (see Fig. 6) due to the compaction of the crushed cells behind the shock front. The above approximations together with Eqs. (1)-(4) lead to the derivations of subsequent Eqs. (5)-(8) following the similar way presented in Ref. (Barnes et al., 2014).

The estimated strain behind the shock front based on Eqs. (1) and (2) is

$\varepsilon_{\mathrm{b}}=\varepsilon_{\mathrm{a}}+\frac{V_{\mathrm{b}}-V_{\mathrm{a}}}{V_{\mathrm{s}}} \approx \frac{V_{\mathrm{i}}}{V_{\mathrm{r}}+S V_{\mathrm{i}}}$ measured from the FE results. For instance, the instantaneous 368 shock nominal strain $\left(\varepsilon_{b}^{\mathrm{i}}\right)$ over the height of the crushed zone, 369 linear fitting leads to

The material state behind the shock front also can be directly 367 

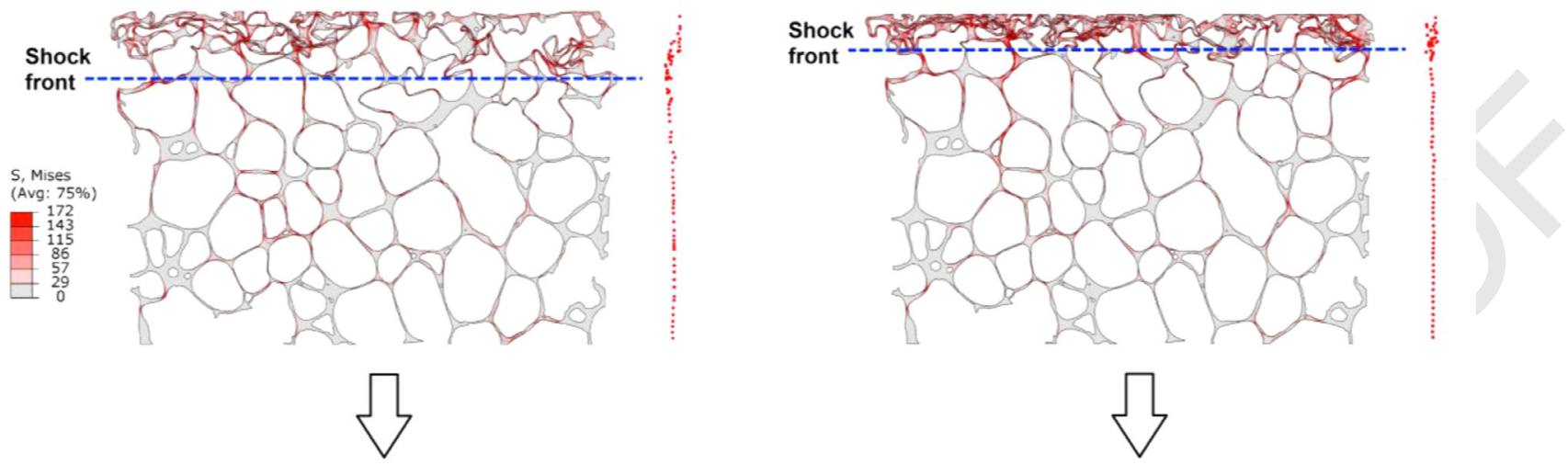

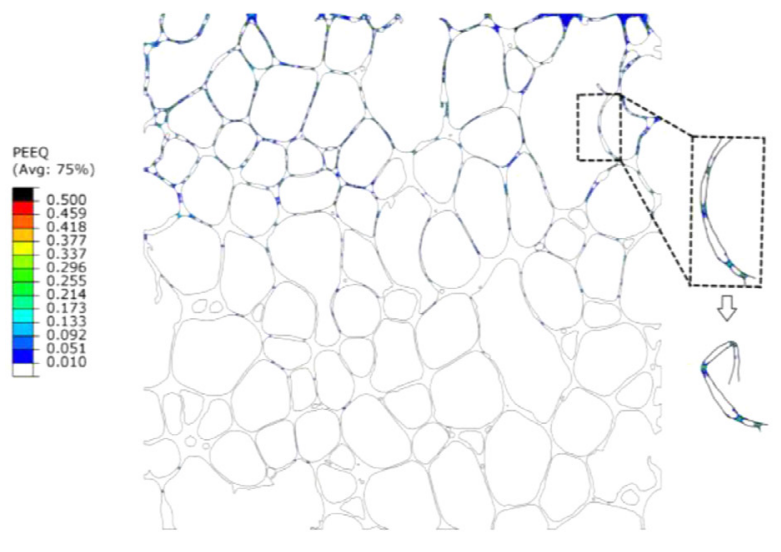

(a) Impact speed $=60 \mathrm{~m} / \mathrm{s}$

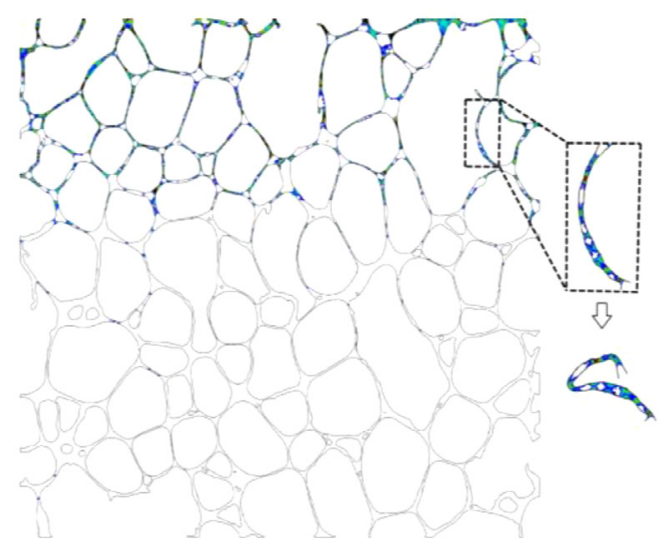

(b) Impact speed $=120 \mathrm{~m} / \mathrm{s}$

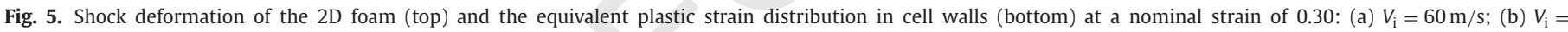

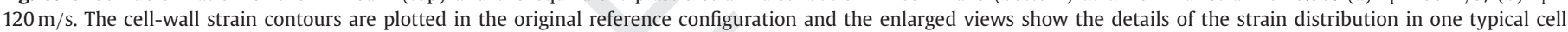
wall with its deformed configuration indicated by the arrow.

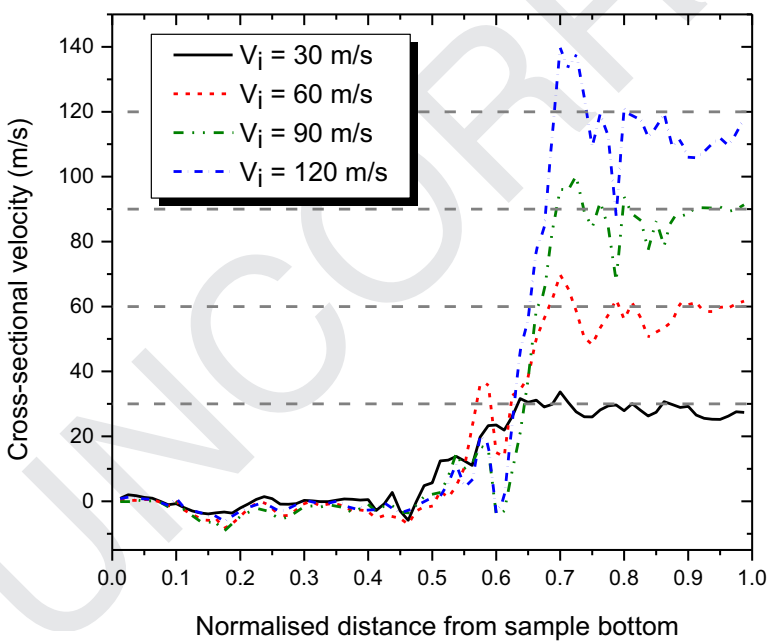

Fig. 6. Distributions of the material velocity $(\varepsilon=0.30)$ averaged over the crossby the original sample height. sections defined in the original reference configuration. The distance is normalised is determined by $\left(H_{\mathrm{b}}-h_{\mathrm{b}}\right) / H_{\mathrm{b}} \approx\left(H-h_{\mathrm{a}}-h_{\mathrm{b}}\right) /\left(H-h_{\mathrm{a}}\right)$ when $H_{\mathrm{a}} \approx h_{\mathrm{a}}$ due to the negligible deformation ahead of the shock front, where $H$ is the initial sample height, $h_{\mathrm{b}}$ and $h_{\mathrm{a}}$ are the heights of the crushed and uncrushed zones measured in the current configuration, respectively. To eliminate the data scatter caused by either the heterogeneity of the cell structure or the numerical algorithm, the shock nominal strain $\left(\varepsilon_{\mathrm{b}}\right.$, or simply shock strain) in the crushed zone is obtained as the average over a series of measurements up to a global nominal strain of 0.40 (equivalent to a certain period of time during the impact). The determinations of other state variables described later follow the same method. Accordingly, the error bars of the data presented in the following figures indicate the standard deviation of the measurement at different global nominal strains.

Fig. 9a shows the comparison between the $\varepsilon_{\mathrm{b}}$ estimated by Eq. (5) and that determined from the FE results in a way described above. A good agreement is achieved and the errors are mainly caused by the limited accuracy to determine the position of the shock front. Also, in Fig. 9a the shock strain $\varepsilon_{\mathrm{b}}$ is compared with the quasi-static densification strain $\varepsilon_{\mathrm{d}}^{\mathrm{qs}}=0.46$ which is determined using an energy absorption efficiency method (Li et al., 2006; Tan et al., 2005a). It reveals that $\varepsilon_{\mathrm{b}}$ at high speed impact is significantly larger than $\varepsilon_{d}^{\mathrm{qs}}$. This issue has been also addressed in recent experimental tests for open-cell aluminium foam (Barnes et al., 2014) and numerical simulations for 3D Voronoi foam (Zheng et al., 2014).

The shock stress (i.e. stress behind the shock front) can be approximated as

$\sigma_{\mathrm{b}}=\sigma_{\mathrm{a}}+\rho_{0} V_{\mathrm{s}}\left(V_{\mathrm{b}}-V_{\mathrm{a}}\right) \approx \sigma_{\mathrm{c}}^{\mathrm{qs}}+\rho_{0} V_{\mathrm{i}}\left(V_{\mathrm{r}}+S V_{\mathrm{i}}\right)$

where the approximations that $V_{\mathrm{a}} \approx 0 \mathrm{~m} / \mathrm{s}, V_{\mathrm{b}} \approx V_{\mathrm{i}}$ and $\sigma_{a} \approx 398$ $\sigma_{c}^{q s}\left(\sigma_{\mathrm{c}}^{\mathrm{qs}}=0.5 \mathrm{MPa}\right.$, the quasi-static collapse stress) are adopted. 399 The approximation of $\sigma_{\mathrm{a}} \approx \sigma_{\mathrm{c}}^{\mathrm{qs}}$ is acceptable as verified by the 400 shock supporting stress and quasi-static stress shown in Fig. 4, although the averaged $\sigma_{\mathrm{a}}$ appears slightly smaller than $\sigma_{\mathrm{c}}^{\mathrm{qs}}$, as also found in previous simulations for 2D Voronoi cellular structures (Liao et al., 2013). It should be noted that in the FE measurement 

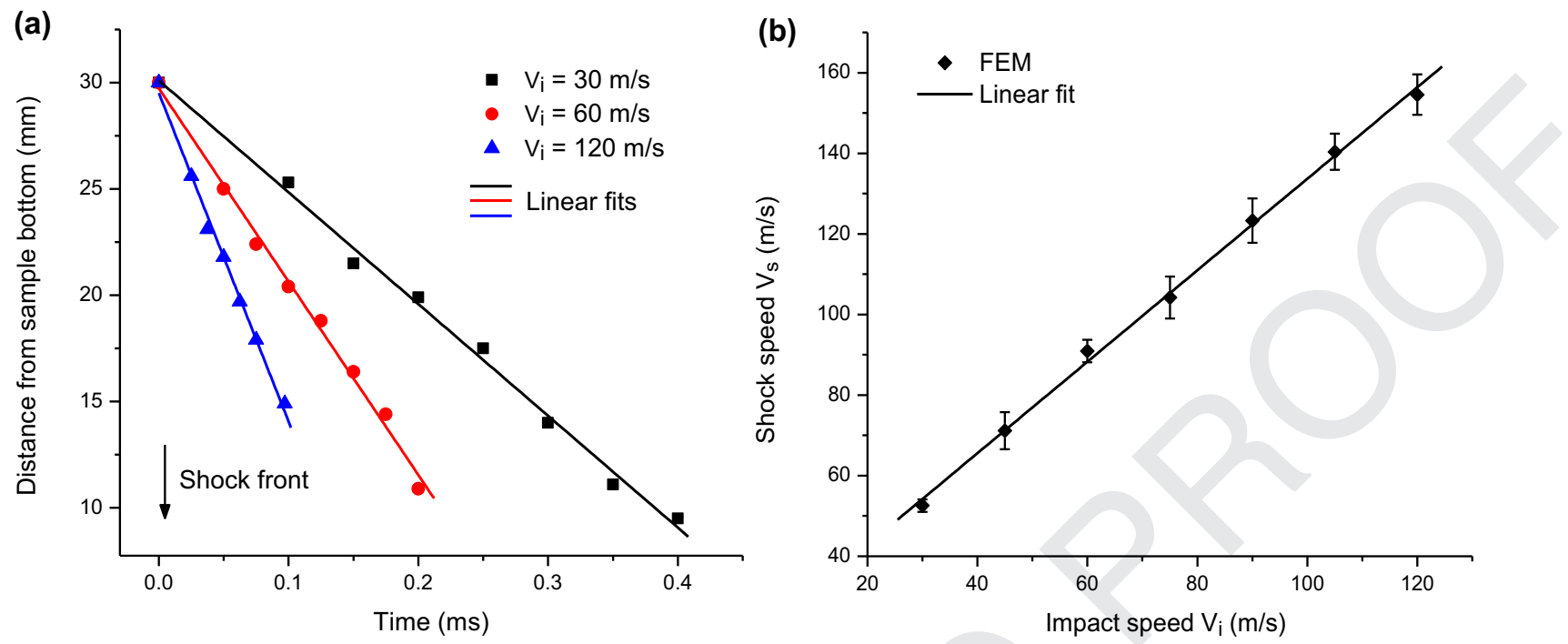

Fig. 7. (a) The variation of the position of shock front with loading time at different impact speeds; (b) the variation of shock speed with impact speed.

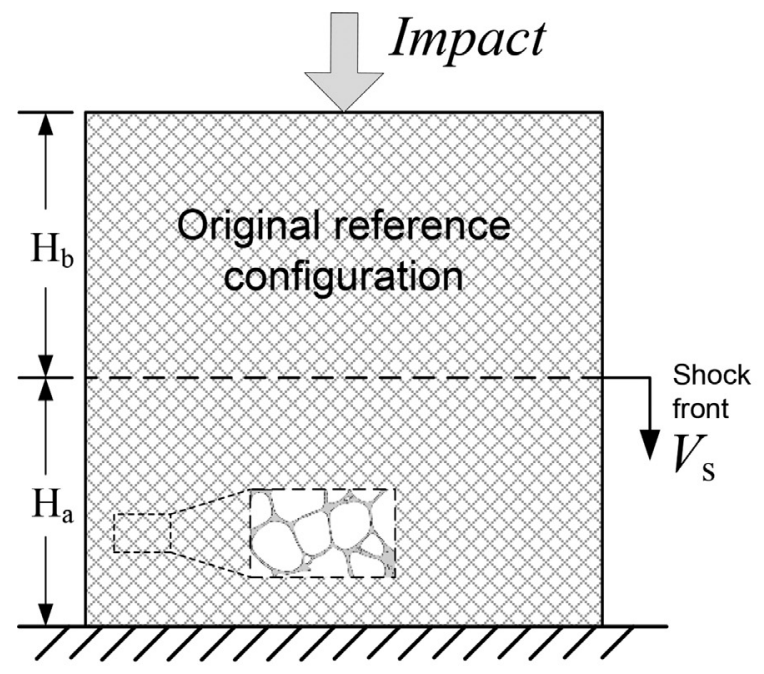

(a)

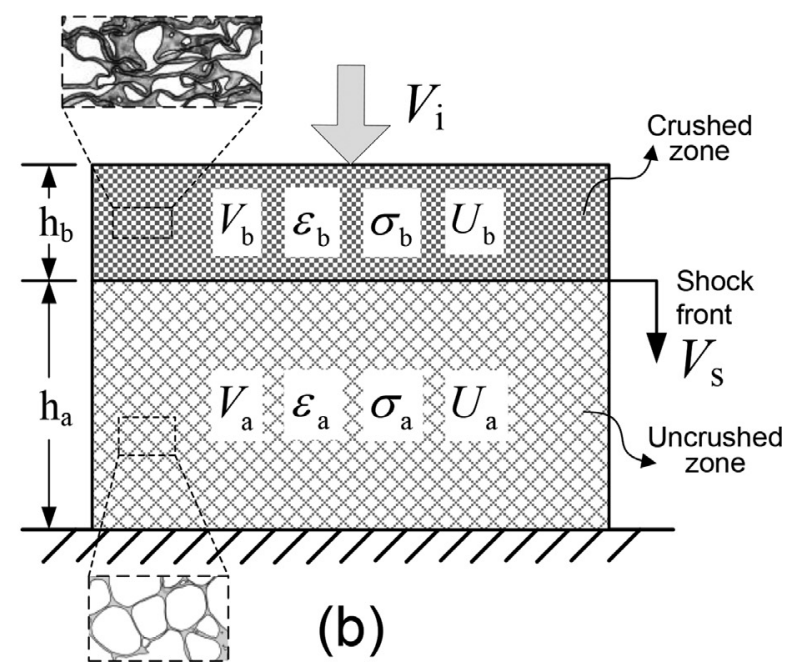

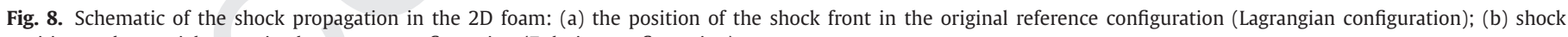
position and material states in the current configuration (Eulerian configuration).

the stresses $\sigma_{\mathrm{a}}$ and $\sigma_{\mathrm{b}}$ are obtained by the averaged reaction forces at rigid platens divided by the original sample width, since both material velocities in the crushed and uncrushed zones are almost constant, as shown in Fig. 6. It should be also noted that in a recent FE analysis, Zheng et al. (2014) used a similar method to obtain $\sigma_{\mathrm{b}}$, while they did not directly measure $\sigma_{\mathrm{a}}$ but instead they derived $\sigma_{\mathrm{a}}$ from Eq. (3), which led to a conclusion that $\sigma_{\mathrm{a}}$ is larger than $\sigma_{\mathrm{c}}^{\mathrm{qc}}$. In fact, the value of $\sigma_{\mathrm{a}}$ depends on the location at which the material is considered as "immediately ahead of shock front" since there is a meso-scale continuous drop of stress from the impact end to the support end. As it still lacks a strict definition of the shock front boundary for heterogeneous cell structure, the $\sigma_{\mathrm{a}}$ and $\sigma_{\mathrm{b}}$ obtained here are based on the measurements on the support and impact ends of the sample, respectively. In Fig. 9b, the $\sigma_{\mathrm{b}}$ estimated by Eq. (6) and that obtained from the FE result is also compared, which shows a reasonable agreement. According to the $\sigma_{\mathrm{b}} / \sigma_{\mathrm{c}}^{\mathrm{qs}}$ shown in Fig. $9 \mathrm{~b}$, the shock stress is significantly larger than the quasi-static collapse stress and increases with impact speed.
Using Eqs. (5) and (6), the strain energy density of the material 424 behind the shock front can be expressed in terms of impact speed, 425 i.e.

$U_{\mathrm{b}}=U_{\mathrm{a}}+\frac{1}{2}\left(\sigma_{\mathrm{b}}+\sigma_{\mathrm{a}}\right)\left(\varepsilon_{\mathrm{b}}-\varepsilon_{\mathrm{a}}\right) \approx \frac{\sigma_{\mathrm{c}}^{\mathrm{q} S} V_{\mathrm{i}}}{V_{\mathrm{r}}+S V_{\mathrm{i}}}+\frac{1}{2} \rho_{0} V_{\mathrm{i}}^{2}$

The prediction from Eq. (7) also compares well with the FE re- 427 sult, as shown in Fig. 10. Furthermore, it is seen that the energy 428 absorption is significantly enhanced at high speed impact. How- 429 ever, such enhancement is not due to the intrinsic rate depen- 430 dence of foams as suggested by Radford et al. (2005), since in 431 the FE model a rate-independent perfect plasticity material model 432 was adopted. A check on the internal energy calculated by Abaqus confirms that the plastic dissipation is dominant and the artificial viscosity in explicit FE simulations (Fleck and Deshpande, 2005) is negligible. This indicates that the enhanced energy absorption capacity results from the increased cell compaction under shock compression which produces more plastic hinges in the cell walls with increasing impact speed, as shown in Fig. 5.

.


(a)
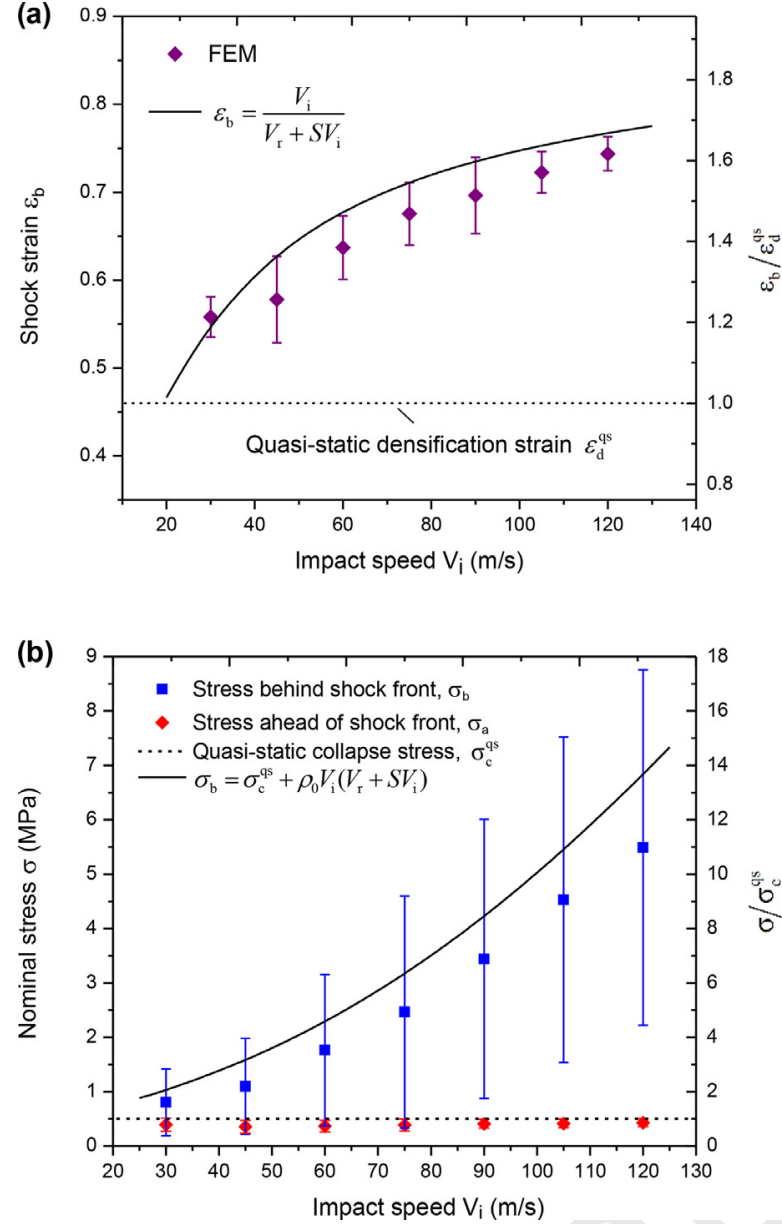

Fig. 9. Variations of material states with impact speed: (a) local nominal strain behind the shock front; (b) nominal stresses ahead of and behind the shock front

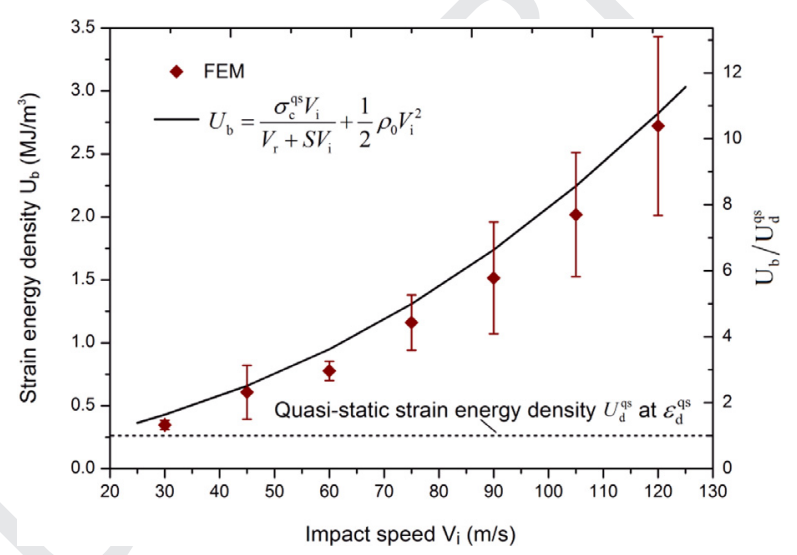

Fig. 10. Strain energy density of the material behind the shock front at different impact speeds.

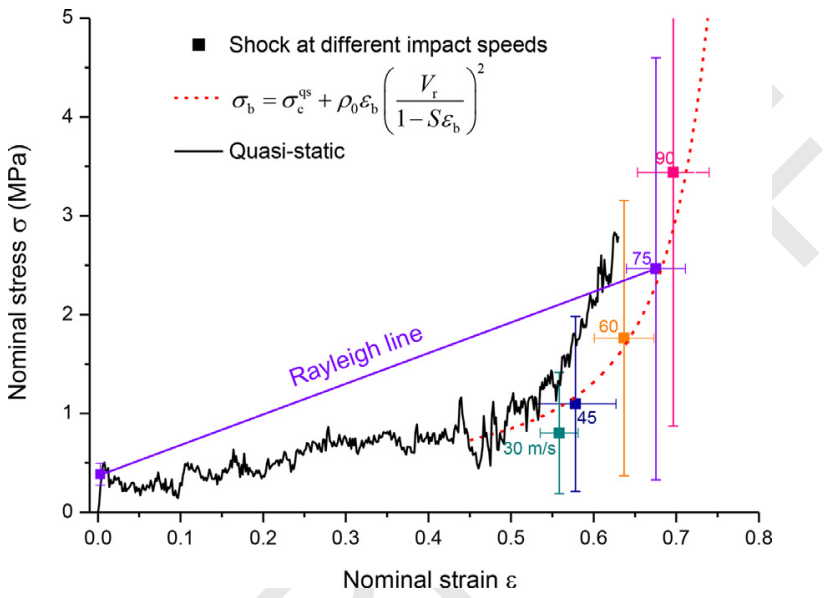

Fig. 11. Comparison between the $\sigma_{\mathrm{b}}-\varepsilon_{\mathrm{b}}$ Hugoniot relation and the quasi-static stress-strain relation. Note that the material states across the shock front are connected by the Rayleigh line.

stress and strain which is often used as a "constitutive relation". It 448 is straightforward to derive the $\sigma_{\mathrm{b}}-\varepsilon_{\mathrm{b}}$ Hugoniot relation by elim- 449 inating $V_{\mathrm{i}}$ in Eqs. (5) and (6), i.e.

$\sigma_{\mathrm{b}} \approx \sigma_{\mathrm{c}}^{\mathrm{qs}}+\rho_{0} \varepsilon_{\mathrm{b}}\left(\frac{V_{\mathrm{r}}}{1-S \varepsilon_{\mathrm{b}}}\right)^{2}$

The prediction of the above equation captures the FE stressstrain states at different impact speeds, as shown in Fig. 11. Under shock compression the material states across the shock front are connected by the Rayleigh line. In other words, the stress jumps when the shock front passes and it does not follow the path defined by the quasi-static stress-strain curve. Moreover, it is evident that the $\sigma_{\mathrm{b}}-\varepsilon_{\mathrm{b}}$ Hugoniot curve is markedly different from the quasi-static stress-strain curve, which confirms the recent observations for 2D Voronoi foam (Liao et al., 2013), 3D Voronoi foam (Zheng et al., 2014) and open-cell aluminium foam (Barnes et al., 2014). This fundamental difference suggests that it is not really appropriate to use a quasi-static stress-strain relation to determine the shock state quantities as done in many previous studies. This needs to be realised and addressed as the quasi-static stress-strain relation is still widely used in shock analysis, e.g. Refs. (Karagiozova and Alves, 2014; Zheng et al., 2016). On the other hand, it should be noted that the shock stress-strain relation applies to different impact speeds as long as shock is initiated, in contrast to the strain-rate effects at intermediate loading speeds which change the stress-strain curve at different strainrates (Zheng et al., 2014).

One may suggest that the $\sigma_{\mathrm{b}}-\varepsilon_{\mathrm{b}}$ Hugoniot relation can serve as a unique dynamic "constitutive stress-strain relation", as done by Zheng et al. (2014). In such a treatment, other Hugoniot relations, including the $V_{s}-V_{i}$ one, can be derived from this dynamic stress-strain relation in conjunction with the conservation laws, see for example the derivation presented in Appendix. However, we notice that to describe this dynamic relation an empirical nonlinear equation is usually needed, the form of which may not be unique to achieve a satisfactory data fitting. In the recent FE study by Zheng et al. (2014) an empirical equation (Eq. 14 in (Zheng et al., 2014)) having similar strain terms to Eq. (8) is proposed to describe the stress-strain states for 3D Voronoi foam under shock compression. It appears that the function selection in Zheng et al. (2014) for stress-strain relation is not unique. In contrast, as long as a unique linear $V_{s}-V_{i}$ relation exists, the form of the stress-strain relation is naturally determined, which seems more logic and reduces uncertainties. Therefore, we recommend the establishment of the "constitutive relation" through measuring 
$V_{\mathrm{s}}-V_{\mathrm{i}}$, as done in present numerical study and previous experimental study (Barnes et al., 2014).

In addition, from Eq. (8) the stress wave speed can be derived as follows

$C_{s}=\sqrt{\frac{\partial \sigma_{\mathrm{b}} / \partial \varepsilon_{\mathrm{b}}}{\rho_{0}}}=\sqrt{\frac{1+S \varepsilon_{\mathrm{b}}}{1-S \varepsilon_{\mathrm{b}}}} V_{\mathrm{r}}$

Accordingly, the derivative of the stress wave speed is obtained as

$\frac{\mathrm{d} C_{\mathrm{s}}}{\mathrm{d} \varepsilon_{\mathrm{b}}}=\frac{S\left(2+S \varepsilon_{\mathrm{b}}\right)}{\left(1-S \varepsilon_{\mathrm{b}}\right)^{2} \sqrt{1-S^{2} \varepsilon_{\mathrm{b}}^{2}}} V_{\mathrm{r}}$

which is constantly greater than zero. This implies that the stress wave speed increases with the increase of strain, which satisfies the requirement of the formation of shock wave in a material exhibiting nonlinear stress-strain relation (Wang, 2007). Therefore, the linear $V_{\mathrm{s}}-V_{\mathrm{i}}$ relation can naturally meet the necessary condition for the formation of shock wave and thus seems more fundamental to characterise shock behaviour in cellular solids.

Similar linear $V_{s}-V_{i}$ Hugoniot relations have also been observed for condensed solids (Davison, 2008; Meyers, 1994), porous materials (Morris, 1991), polystyrene foams (Morris, 1991) and open-cell aluminium foams (Barnes et al., 2014). For condensed solids the constant $V_{\mathrm{r}}$ in Eq. (1) is normally close to the speed of sound (Davison, 2008; Meyers, 1994), but it is not applicable to the 2D foam studied here or open-cell aluminium foam (Barnes et al., 2014). The sound speed $C_{0}$ for the 2D foam, which is measured as the ratio of the initial sample height to the time duration before the stress at the support end becomes non-zero, is about $4010 \mathrm{~m} / \mathrm{s}$, while the calculated sound speed of Alporas foam is about $1720 \mathrm{~m} / \mathrm{s}$ based on its overall Young's modulus and density. Both are far larger than $V_{\mathrm{r}}$. This represents a fundamental difference in the shock physics between cellular solids and condensed solids. As the coefficient $S$ is close to one for cellular solids, the $V_{\mathrm{r}}$ indicates the difference between shock speed and impact speed (also material speed in the crushed zone according to Fig. 6). The consequence of a small $V_{\mathrm{r}}$ is that the shock speed is close to material speed in cellular solids. This contrasts with most of condensed solids (e.g. aluminium) wherein shock wave travels much faster than material points (Meyers, 1994). Such a difference arises from the cell structural response nature of the shock in cellular solids, i.e. the macroscopic discontinuity across shock front is associated with the local densification of the cells, rather than atomic-level mechanisms controlling the shock in condensed solids (Meyers, 1994). Nevertheless, shock response in cellular solids is still governed by macroscopic wave equations, as demonstrated in Section 3.3.

It is also of interest to explore the physical implication of the coefficient $S$ in the linear $V_{\mathrm{s}}-V_{\mathrm{i}}$ relation. It is evident that when $V_{\mathrm{i}} \rightarrow \infty$ the upper limit of $\varepsilon_{\mathrm{b}}$ determined by Eq. (5) is $1 / S=0.881$, which is almost identical to the "full densification", i.e. $1-\mathrm{RD}=0.875$ when lateral deformation is negligible. It confirms that the shock occurring in the 2D foam is dominated by the structural densification, which is intensified when impact speed increases, as shown in Fig. 5. In other words, increasing impact speed produces more plastic hinges in cell walls rather than switching deformation mode to the uniaxial compression of the cell walls. Consequently, the shock strain, stress and energy all increase with impact speed.

\subsection{Critical impact speed for shock initiation}

It is important to know the critical impact speed for shock initiation in cellular solids. The determination of the critical impact speed often depends on the assumed "constitutive stress-strain relation". For instance, when a rigid, linear hardening plastic, locking
(R-LHP-L) constitutive model is assumed, the critical impact speed is given by (Wang et al., 2013; Zheng et al., 2012)

$V_{\mathrm{c} \_s}=\varepsilon_{\mathrm{d}} \sqrt{\frac{E_{\mathrm{h}}}{\rho_{0}}}$

where $E_{\mathrm{h}}$ is the hardening modulus, $\varepsilon_{\mathrm{d}}$ is the densification strain 550 and $\sigma_{0}$ is the initial density of the cellular material. However, when 551 a rigid, perfectly plastic, locking (R-PP-L) constitutive model is as- 552 sumed, shock will be initiated at any impact speed larger than zero 553 (Wang et al., 2013). This unreasonable conclusion is corrected by 554 considering the elastic precursor wave (Wang et al., 2013) and the 555 threshold of shock enhancement of stress (Ashby et al., 2000; Tan 556 et al., 2005b). For the former correction, the critical impact speed 557 becomes

$V_{\mathrm{c} \_s_{-}}=\varepsilon_{\mathrm{Y}} \sqrt{\frac{E}{\rho_{0}}}$

where $E$ is the elastic modulus and $\varepsilon_{\mathrm{Y}}$ is the yield strain (normally 559 adopted as the collapse strain). For the latter correction, the critical impact speed depends on an empirical parameter $\alpha$, i.e.

$V_{\mathrm{c}_{-} \mathrm{s}}=\sqrt{\alpha \frac{\sigma_{\mathrm{pl}} \varepsilon_{\mathrm{d}}}{\rho_{0}}}$

where $\sigma_{\mathrm{pl}}$ is the plateau stress and $\alpha$ satisfies $\Delta \sigma=\alpha \sigma_{\mathrm{pl}}$ where $\Delta \sigma$ is the stress enhancement due to shock. The plateau stress can be obtained by energy absorption equivalence at the plateau stage (Li et al., 2006; Tan et al., 2005a). The value of $\alpha$ was adopted as 0.1 by Ashby et al. (2000) and 2.0 by Tan et al. (2005b). Although Tan et al. (2005b) claimed that their adoption of $\alpha=2.0$ is based on the so-called kinematic existence condition for continuing "steady-shock" and the prediction agrees with their experimental results, Wang et al. (2013) proved that the theoretical basis of this kinematic existence condition is incorrect and the mistake is caused by the confusion in basic concept between the "energy conservation in an isolate system" and the "energy conservation across a shock wave".

The further improvement of the prediction using a similar approach to that described above may be made by considering a more realistic "constitutive stress-strain relation", such as a nonlinear one. For instance, Zheng et al. (2013) adopted a power law, i.e. $\sigma=\sigma_{0}+K \varepsilon^{n}$ where $\sigma_{0}$ is the yield stress, $K$ is the strength index and $n$ is the strain-hardening index, to describe the quasistatic stress-strain relation, and then gave the following equation to predict the critical impact speed

$V_{\mathrm{c} \_\mathrm{s}}=\varepsilon_{\mathrm{d}}^{(\mathrm{n}+1) / 2} \sqrt{\frac{K}{\rho_{0}}}$

The common problem of above methods for the determina- 583 tion of critical impact speed is that they are based on quasi-static stress-strain relation, which is actually different from that under shock compression. Experimental (Barnes et al., 2014; Gibson and Ashby, 1997; Sun et al., 2014; Tan et al., 2005a) and numerical (Liu et al., 2009; Ruan et al., 2003; Zheng et al., 2005) observations have demonstrated that under quasi-static (including "low" dynamic compressions) the cell crush bands are distributed randomly in terms of their location and orientation, which are sensitive to the structural defects and the complicated transmission of load between neighbouring cells, in contrast to the shock deformation. Consequently, the shock stress-strain relation is distinct from the quasi-static one, as shown in Refs. (Barnes et al., 2014; Liao et al., 2013; Zheng et al., 2014) as well as in Fig. 11. This suggests that a "constitutive relation" without involving shock is not applicable to shock compression, and the prediction of the critical impact speed for shock initiation based on continuum approach 
Table 2

Predictions of the critical impact speed for shock initiation of the 2D foam using different methods (unit: $\mathrm{m} / \mathrm{s}$ ).

\begin{tabular}{lllllll}
\hline FE result & $\frac{V_{\mathrm{r}}}{1 / \varepsilon_{\mathrm{d}}^{\text {sh }}-S}$ (Eq. 15) & $\varepsilon_{\mathrm{d}}^{(\mathrm{n}+1) / 2} \sqrt{\frac{K}{\rho_{0}}}($ Eq. 14) & $\sqrt{0.1 \frac{\sigma_{\mathrm{pl}} \varepsilon_{\mathrm{d}}}{\rho_{0}}}$ (Eq. 13) & $\sqrt{2 \frac{\sigma_{\mathrm{pl}} \varepsilon_{\mathrm{d}}}{\rho_{0}}}$ (Eq. 13) & $\varepsilon_{\mathrm{Y}} \sqrt{\frac{E}{\rho_{0}}}($ Eq. 12) & $\varepsilon_{\mathrm{d}} \sqrt{\frac{E_{\mathrm{h}}}{\rho_{0}}}($ Eq. 11) \\
\hline 24 & 22 & 9 & 38 & 4 & 19 \\
\hline
\end{tabular}

Table 3

Predictions of the critical impact speed for shock initiation of open-cell aluminium foam (Barnes et al., 2014) using different methods (unit: $\mathrm{m} / \mathrm{s}$ ).

\begin{tabular}{lllllll}
\hline Test result & $\frac{V_{\mathrm{r}}}{1 / \varepsilon_{\mathrm{d}}^{\mathrm{sh}}-S}($ Eq. 15) & $\varepsilon_{\mathrm{d}}^{(\mathrm{n}+1) / 2} \sqrt{\frac{K}{\rho_{0}}}($ Eq. 14) & $\sqrt{0.1 \frac{\sigma_{\mathrm{p} \mid \varepsilon_{\mathrm{d}}}}{\rho_{0}}}$ (Eq. 13) & $\sqrt{2 \frac{\sigma_{\mathrm{p} \mid \varepsilon_{\mathrm{d}}}}{\rho_{0}}}$ (Eq. 13) & $\varepsilon_{\mathrm{Y}} \sqrt{\frac{E}{\rho_{0}}}($ Eq. 12) & $\varepsilon_{\mathrm{d}} \sqrt{\frac{E_{\mathrm{h}}}{\rho_{0}}}($ Eq. 11) \\
\hline $40-60$ & 56 & 43 & 21 & 92 & 13 & 38 \\
\hline
\end{tabular}

Table 4

Predictions of the critical impact speed for shock initiation of 3D Voronoi foam (Zheng et al., 2014) using different methods (unit: m/s).

\begin{tabular}{lllllll}
\hline FE result & $\frac{V_{\mathrm{r}}}{1 / \varepsilon_{\mathrm{d}}^{\mathrm{h}}-S}($ Eq. 15) & $\varepsilon_{\mathrm{d}}^{(\mathrm{n}+1) / 2} \sqrt{\frac{K}{\rho_{0}}}$ (Eq. 14) & $\sqrt{0.1 \frac{\sigma_{\mathrm{p} p} \varepsilon_{\mathrm{d}}}{\rho_{0}}}$ (Eq. 13) & $\sqrt{2 \frac{\sigma_{\mathrm{p} 1} \varepsilon_{\mathrm{d}}}{\rho_{0}}}$ (Eq. 13) & $\varepsilon_{\mathrm{Y}} \sqrt{\frac{E}{\rho_{0}}}($ Eq. 12) & $\varepsilon_{\mathrm{d}} \sqrt{\frac{E_{\mathrm{h}}}{\rho_{0}}}($ Eq. 11) \\
\hline 57 & 69 & 40 & 177 & 20 & 78 \\
\hline
\end{tabular}

should take account of the Hugoniot relations, which, however, has not been attempted before.

Eq. (5) gives a relationship between the impact speed and the shock strain behind the shock front. This equation can be used to determine the critical impact speed for the occurrence of the shock compression when the critical shock strain is determined, i.e.

$V_{\mathrm{c} \_s \mathrm{~s}}=\frac{V_{\mathrm{r}}}{1 / \varepsilon_{\mathrm{c} \_s_{-}}-S}$

where $\varepsilon_{c_{-} s}$ is the critical shock strain, $V_{\mathrm{r}}$ and $S$ are parameters in the linear $V_{s}-V_{i}$ Hugoniot relation, namely Eq. (1). A characteristic strain, e.g. lock-up or densification strain, based on quasistatic stress-strain relation has been used previously to determine the critical impact speed, i.e. Eqs. (11), (13) and (14). Here, we adopted $\varepsilon_{\mathrm{c}_{\_} s} \approx \varepsilon_{\mathrm{d}}^{\text {sh }}$ where $\varepsilon_{\mathrm{d}}^{\text {sh }}$ is the shock "densification strain" obtained from Eq. (8) using the energy absorption efficiency method which proves capable of capturing the onset of foam densification (Li et al., 2006; Tan et al., 2005a). According to our simulation results, i.e. $\varepsilon_{\mathrm{d}}^{\text {sh }}=0.49, V_{\mathrm{r}}=20.16 \mathrm{~m} / \mathrm{s}$ and $S=1.135$, we obtained that $V_{c_{\_} s}=22 \mathrm{~m} / \mathrm{s}$, which is close to the critical impact speed $(\sim 24 \mathrm{~m} / \mathrm{s})$ identified directly from a series of FE simulations. We also applied this equation to the experimental results for the open-cell aluminium foam tested by Barnes et al. (2014), and obtained that $V_{\mathrm{c}_{\_} \mathrm{s}}=56 \mathrm{~m} / \mathrm{s}$ which falls in the speed range reported $(40-60 \mathrm{~m} / \mathrm{s})$ wherein the foam compression transits into shock. For 3D Voronoi foam, the critical impact speed is predicted as $69 \mathrm{~m} / \mathrm{s}$ using the material parameters reported in Ref. Zheng et al., 2014), in comparison with the critical impact speed of $57 \mathrm{~m} / \mathrm{s}$ reported therein. It is evident that $V_{\mathrm{r}} /\left(1 / \varepsilon_{\mathrm{d}}^{\mathrm{sh}}-S\right)$ gives a quick and reasonable estimate, and generally outperforms other methods based on quasi-static material parameters, i.e. Eqs. (11)-((14), see the comparison in Tables 2-4. In addition, it is interesting to note that $V_{\mathrm{c}_{-} s}$ is very close to $V_{\mathrm{r}}$ for the 2D foam studied here.

It should be noted that the difference of cell deformations under quasi-static and shock compressions has been reflected in the distinctive shock stress-strain relation used to estimate the critical shock strain. For shock compression, the critical shock strain corresponding to the onset of the collective cell densification behind the shock front. This is consistent with the shock deformation mechanism, as shown in Fig. 5.

\section{Conclusions}

Based on a cross-sectional geometry obtained from X-ray CT image of a closed-cell aluminium Alporas foam sample, a 2D image-based FE model is developed, for the first time of this type, to study the shock compression of cellular solids. This study leads to the following conclusions:

1 The shock compression occurs above a critical impact speed which can be estimated by shock Hugoniot relations, and under shock compression, the compaction in crushed cells and the plastic deformation in cell walls are enhanced when impact speed increases, leading to the nonlinear increase of shock strain, stress and energy with impact speed;

2 A linear shock Hugoniot relation between shock speed and impact speed is obtained, of which the constant term is much lower than sound speed and implies the local structural densification of cells, in contrast to the constant term close to sound speed controlled by atomic-level mechanisms in condensed solids;

3 Other shock state variables derived from the linear shock Hugoniot relation and conservation laws in continuum mechanics are in good agreement with the direct FE measurement, and the measurement of shock speed provides sufficient information for understanding the shock behaviour;

4 The unique linear Hugoniot relation is preferential to characterise the shock constitutive relation as it avoids the uncertainty about the selection of a nonlinear function for the shock "constitutive stress-strain relation" which is distinct from the quasi-static one;

5 A new analytical method based on the linear Hugoniot relation is proposed to estimate the critical impact speed for shock initiation, which is verified by the present FE simulations and previous experimental and numerical results, and outperforms the existing methods.

\section{Acknowledgements}

The authors would like to acknowledge the use of Computational Shared Facility at The University of Manchester (UoM). In addition, the first author is grateful for the research scholarship from UoM and for the imaging technical support from $\mathrm{Dr} \mathrm{T}$. Lowe and Dr R. Bradley. The first author also appreciates the discussion with Mr Y. Ding, Prof Z. Zheng, Prof L.-L. Wang and Prof S. Kyriakides on the shock behaviour of foams. The Manchester X-ray Imaging Facility was funded in part by the EPSRC (grants EP/F007906/1, EP/F001452/1 and EP/I02249X/1). The second author, as an adjunct professor at Beijing Institute of Technology, acknowledges the 
693

$$
\frac{1}{\varepsilon_{\mathrm{B}}}=1+\frac{\sqrt{D / \rho_{0}}}{v}
$$

\section{According to Eq. (19) in Ref. (Zheng et al., 2014), we have}

$\dot{\Phi}=v / \varepsilon_{\mathrm{B}}$

support from State Key Laboratory of Explosion Science and Technology (ZDKT11-03).

In Ref. (Zheng et al., 2014), the dynamic stress-strain relation is $\sigma=\sigma_{0}^{\mathrm{d}}+D \varepsilon /(1-\varepsilon)^{2}$

where $\dot{\Phi}$ is the shock speed. Combining Eq. (A.3) with Eq. (A.4), a linear relation between shock speed and impact speed is obtained as follows

$\dot{\Phi}=v+\sqrt{D / \rho_{0}}$

\section{References}

Ashby, M.F., Evans, A.G., Fleck, N.A., Gibson, L.J., Hutchinson, J.W., Wadley, H.N.G., 2000. Metal Foams: A Design Guide. Elsevier.

Barnes, A.T., Ravi-Chandar, K., Kyriakides, S., Gaitanaros, S., 2014. Dynamic crushing of aluminum foams: Part I - Experiments. Int. J. Solids Struct. 51, 1631-1645.

Chen, C., Lu, T.J., Fleck, N.A., 1999. Effect of imperfections on the yielding of twodimensional foams. J. Mech. Phys. Solids 47, 2235-2272.

Davison, L., 2008. Fundamentals of Shock Wave Propagation in Solids. Springer.

Elnasri, I., Pattofatto, S., Zhao, H., Tsitsiris, H., Hild, F., Girard, Y., 2007. Shock enhancement of cellular structures under impact loading: part I experiments. J. Mech. Phys. Solids 55, 2652-2671.

Fleck, N.A., Deshpande, V.S., 2005. Closure to "discussion of 'the resistance of clamped sandwich beams to shock loading' " (2005, ASME J. Appl. Mech., [bold 72], pp. 978-979). J. Appl. Mech. 72, 980.

Gibson, L.J., Ashby, M.F., 1997. Cellular Solids: Structure and Properties, second ed. Cambridge University Press.

Hildebrand, T., Rüegsegger, P., 1997. A new method for the model-independent assessment of thickness in three-dimensional images. Journal of Microscopy 185 67-75.

Hönig, A., Stronge, W.J., 2002. In-plane dynamic crushing of honeycomb. Part II: application to impact. Int. J. Mech. Sci. 44, 1697-1714.

Jeon, I., Asahina, T., Kang, K.-J., Im, S., Lu, T.J., 2010. Finite element simulation of the plastic collapse of closed-cell aluminum foams with X-ray computed tomography. Mech. Mater. 42, 227-236.

Karagiozova, D., Alves, M., 2014. Compaction of a double-layered metal foam block impacting a rigid wall. Int. J. Solids Struct. 51, 2424-2438.

Karagiozova, D., Langdon, G.S., Nurick, G.N., 2012. Propagation of compaction waves in metal foams exhibiting strain hardening. Int. J. Solids Struct. 49, 2763-2777.

Li, K., Gao, X.L., Wang, J., 2007. Dynamic crushing behavior of honeycomb structures with irregular cell shapes and non-uniform cell wall thickness. Int. J. Solids Struct. 44, 5003-5026.

Li, Q.M., Magkiriadis, I., Harrigan, J.J., 2006. Compressive Strain at the Onset of Densification of Cellular Solids. Journal of Cellular Plastics 42, 371-392.

Li, Q.M., Meng, H., 2002. Attenuation or enhancement - A one-dimensional analysis on shock transmission in the solid phase of a cellular material. Int. J. Impact Eng. 27, 1049-1065.

Li, Q.M., Reid, S.R., 2006. About one-dimensional shock propagation in a cellular material. Int. J. Impact Eng. 32, 1898-1906.

Liao, S., Zheng, Z., Yu, J., 2013. Dynamic crushing of 2D cellular structures: Local strain field and shock wave velocity. Int. J. Impact Eng. 57, 7-16.
Liu, Y., Zhang, X.-C., 2009. The influence of cell micro-topology on the in-plane dynamic crushing of honeycombs. Int. J. Impact Eng. 36, 98-109.

Liu, Y.D., Yu, J.L., Zheng, Z.J., Li, J.R., 2009. A numerical study on the rate sensitivity of cellular metals. Int. J. Solids Struct. 46, 3988-3998.

Ma, G.W., Ye, Z.Q., Shao, Z.S., 2009. Modeling loading rate effect on crushing stress of metallic cellular materials. Int. J. Impact Eng. 36, 775-782.

Maire, E., Withers, P.J., 2014. Quantitative X-ray tomography. Int. Mater. Rev. 59, 143.

McDonald, S.A., Dedreuil-Monet, G., Yao, Y.T., Alderson, A., Withers, P.J., 2011. In situ 3D X-ray microtomography study comparing auxetic and non-auxetic polymeric foams under tension. Phys. Status Solidi (B) 248, 45-51.

McDonald, S.A., Mummery, P.M., Johnson, G., Withers, P.J., 2006. Characterization of the three-dimensional structure of a metallic foam during compressive deformation. J. Microsc. 223, 150-158.

Meyers, M.A., 1994. Dynamic Behavior of Materials. John Wiley \& Sons.

Morris, C.E., 1991. Shock-wave equation-of-state studies at Los Alamos. Shock Waves $1,213-222$.

Pattofatto, S., Elnasri, I, Zhao, H., Tsitsiris, H., Hild, F., Girard, Y, 2007. Shock enhancement of cellular structures under impact loading: Part II analysis. J. Mech. Phys. Solids 55, 2672-2686.

Radford, D.D., Deshpande, V.S., Fleck, N.A., 2005. The use of metal foam projectiles to simulate shock loading on a structure. Int. J. Impact Eng. 31, 1152-1171.

Reid, S.R., Peng, C., 1997. Dynamic uniaxial crushing of wood. Int. J. Impact Eng. 19, $531-570$.

Ruan, D., Lu, G., Wang, B., Yu, T.X., 2003. In-plane dynamic crushing of honeycombs-a finite element study. Int. J. Impact Eng. 28, 161-182.

Simone, A.E., Gibson, L.J., 1998. Aluminum foams produced by liquid-state processes. Acta Materialia 46, 3109-3123.

Simone, A.E., Gibson, L.J., 1998. The effects of cell face curvature and corrugations on the stiffness and strength of metallic foams. Acta Materialia 46, 3929-3935.

Simone, A.E., Gibson, L.J., 1998. Effects of solid distribution on the stiffness and strength of metallic foams. Acta Materialia 46, 2139-2150.

Sun, Y., Amirrasouli, B., Razavi, S.B., Li, Q.M., Lowe, T., Withers, P.J. , 2016. The variation in elastic modulus throughout the compression of foam materials. Acta Materialia ).

Sun, Y., Li, Q.M., 2015. Effect of entrapped gas on the dynamic compressive behaviour of cellular solids. Int. J. Solids Struct. 63, 50-67.

Sun, Y, Li, Q M. Lowe, T, McDonald, S.A., Withers, PJ. 2016. Investigation of strainrate effect on the compressive behaviour of closed-cell aluminium foam by 3D image-based modelling. Mater. Des. 89, 215-224.

Sun, Y.L., Lowe, T., McDonald, S.A., Li, Q.M., Withers, P.J., 2014. In Situ Investigation and Image-Based Modelling of Aluminium Foam Compression Using Micro X-Ray Computed Tomography, Visual Computing: Scientific Visualization and Imaging Systems. Springer, pp. 189-197.

Tan, P.J., Reid, S.R., Harrigan, J.J., 2012. On the dynamic mechanical properties of open-cell metal foams - a re-assessment of the 'simple-shock theory'. Int. J. Solids Struct. 49, 2744-2753.

Tan, P.J., Reid, S.R., Harrigan, J.J., Zou, Z., Li, S., 2005. Dynamic compressive strength properties of aluminium foams. Part I-experimental data and observations. Journal of the Mechanics and Physics of Solids 53, 2174-2205.

Tan, P.J., Reid, S.R., Harrigan, J.J., Zou, Z., Li, S., 2005. Dynamic compressive strength properties of aluminium foams. Part II-'shock' theory and comparison with experimental data and numerical models. J. Mech. Phys. Solids 53, 2206-2230.

Wang, L.-L., Yang, L.-M., Ding, Y.-Y. , 2013. On the energy conservation and critical velocities for the propagation of a "steady-shock" wave in a bar made of cellular material. Acta Mechanica Sinica 1-9.

Wang, L., 2007. Foundations of Stress Waves. Elsevier Science Ltd, Amsterdam.

Young, P.G., Beresford-West, T.B.H., Coward, S.R.L., Notarberardino, B., Walker, B., Abdul-Aziz, A., 2008. An efficient approach to converting three-dimensional image data into highly accurate computational models. Philos. Trans. R. Soc. A Math. Phys. Eng. Sci. 366, 3155-3173.

Zhang, X.-C., Liu, Y., Wang, B., Zhang, Z.-M., 2010. Effects of defects on the in-plane dynamic crushing of metal honeycombs. Int. J. Mech. Sci. 52, 1290-1298.

Zheng, J., Qin, Q., Wang, T.J., 2016. Impact plastic crushing and design of densitygraded cellular materials. Mech. Mater. 94, 66-78.

Zheng, Z., Liu, Y., Yu, J., Reid, S.R., 2012. Dynamic crushing of cellular materials: Continuum-based wave models for the transitional and shock modes. Int. J. Impact Eng. 42, 66-79.

Zheng, Z., Wang, C., Yu, J., Reid, S.R., Harrigan, J.J., 2014. Dynamic stress-strain states for metal foams using a 3D cellular model. J. Mech. Phys. Solids 72, 93-114.

Zheng, Z., Yu, J., Li, J., 2005. Dynamic crushing of 2D cellular structures: a finite element study. Int. J. Impact Eng. 32, 650-664.

Zheng, Z., Yu, J., Wang, C., Liao, S., Liu, Y., 2013. Dynamic crushing of cellular materials: a unified framework of plastic shock wave models. Int. J. Impact Eng. 53, 29-43.

Zou, Z., Reid, S.R., Tan, P.J., Li, S., Harrigan, J.J., 2009. Dynamic crushing of honeycombs and features of shock fronts. Int. J. Impact Eng. 36, 165-176. 